\title{
Solving the Laplace equation by meshless collocation using harmonic kernels
}

\section{Robert Schaback}

Received: 28 June 2007 / Accepted: 30 March 2008 / Published online: 24 May 2008

(C) The Author(s) 2008

\begin{abstract}
We present a meshless technique which can be seen as an alternative to the method of fundamental solutions (MFS). It calculates homogeneous solutions of the Laplacian (i.e. harmonic functions) for given boundary data by a direct collocation technique on the boundary using kernels which are harmonic in two variables. In contrast to the MFS, there is no artificial boundary needed, and there is a fairly general and complete error analysis using standard techniques from meshless methods for the recovery of functions. We present two explicit examples of harmonic kernels, a mathematical analysis providing error bounds and convergence rates, and some illustrating numerical examples.
\end{abstract}

Keywords Laplace equation • Meshless collocation · Harmonic kernels

\section{Introduction}

The method of fundamental solutions (MFS) solves a homogeneous boundary value problem, for example a Dirichlet problem

$$
\begin{aligned}
-\Delta u & =0 \text { in } \Omega \\
u & =f \text { in } \partial \Omega
\end{aligned}
$$

Communicated by Yuesheng Xu.

R. Schaback $(\varangle)$

Institut fuer Numerische und Angewandte Mathematik (NAM),

Georg-August-Universität Göttingen, Lotzestrasse 16-18, 37073,

Göttingen, Germany

e-mail: schaback@math.uni-goettingen.de 
for the Laplace equation via approximation of the boundary data by traces of fundamental solutions centered at source points outside the domain in question. The method has been used extensively in recent years, and there are excellent surveys [1-3]. However, the method has two drawbacks:

1. It lacks a general error analysis, since the existing mathematical results are confined to concentric circles as true and "fictitious" boundaries,

2. It needs source points outside the domain which are not easy to place properly.

This contribution proceeds differently by recurring to standard kernel-based reconstruction of functions from scattered data.

We consider a domain $\Omega$ given in boundary-parameterized polar form

$$
\begin{aligned}
\partial \Omega & :=\left\{x \in \mathbb{R}^{2}: x=\mathcal{R}(t), t \in[0,2 \pi]\right\} \\
\mathcal{R} & :[0,2 \pi] \rightarrow I R, 2 \pi \text {-periodic curve, } \\
\mathcal{R}(t) & =\rho(t)(\cos (t), \sin (t)), t \in[0,2 \pi]
\end{aligned}
$$

and we assume that the domain is bounded by

$$
0<\rho(t) \leq R<\infty \text { for all } t \in[0,2 \pi]
$$

Furthermore, symmetric and positive definite harmonic kernels are constructed on $I R^{2} \times I R^{2}$. If $K$ is such a kernel, there are harmonic trial functions given by

$$
s(x):=\sum_{j=1}^{N} a_{j} K\left(x, x_{j}\right), x \in \Omega
$$

for any set $X:=\left\{x_{1}, \ldots, x_{N}\right\}$ of $N$ pairwise distinct points

$$
x_{j}=\mathcal{R}\left(t_{j}\right)=\rho\left(t_{j}\right)\left(\cos \left(t_{j}\right), \sin \left(t_{j}\right)\right), t_{j} \in[0,2 \pi], 1 \leq j \leq N,
$$

on the boundary $\partial \Omega$ of $\Omega$ and arbitrary vectors $a=\left(a_{1}, \ldots, a_{N}\right)^{T} \in \mathbb{I R}^{N}$.

If the kernel is positive definite (see e.g. [8] for details on kernels and their applications), one can collocate a given function $f: \partial \Omega \rightarrow I R$ on the boundary points by solving the system

$$
\sum_{j=1}^{N} a_{j} K\left(x_{k}, x_{j}\right)=f\left(x_{k}\right), 1 \leq k \leq N
$$

Then one evaluates the boundary error and uses the maximum principle to have an error bound

$$
\|f-s\|_{\infty, \bar{\Omega}} \leq\|f-s\|_{\infty, \partial \Omega} .
$$


Thus the main problem is to guarantee a small boundary error via the collocation. The error can be rewritten as a periodic function via

$$
\begin{aligned}
f(\mathcal{R}(t))-s(\mathcal{R}(t)) & =f(\mathcal{R}(t))-\sum_{j=1}^{N} a_{j} K\left(\mathcal{R}(t), \mathcal{R}\left(t_{j}\right)\right) \\
\tilde{f}(t)-\tilde{s}(t) & =\tilde{f}(t)-\sum_{j=1}^{N} a_{j} \tilde{K}\left(t, t_{j}\right)
\end{aligned}
$$

where we used the tilde to denote the periodicized functions.

From this point on, one can forget the original setting and consider the problem of recovering the periodic function $\tilde{f}$ from data using the periodic kernel $\tilde{K}$. For this, a well-established theory is available (see e.g. [10]), but we shall have to establish the necessary conditions for its applicability. It results in error bounds which are tightly connected to the smoothness of $\tilde{f}$ and $\tilde{K}$.

\section{General harmonic kernels}

The most convenient source for harmonic functions in 2D are the real or imaginary parts of complex analytic functions. We shall exploit this by writing a complex variable

$$
z=x+i y=r(\cos (\varphi)+i \sin (\varphi))
$$

in polar coordinates $(r, \varphi)$. The easiest case arises from

$$
z^{n}=r^{n}(\cos (n \varphi)+i \sin (n \varphi))
$$

and yields harmonic polynomials

$$
r^{n} \cos (n \varphi) \text { and } r^{n} \sin (n \varphi), n \geq 0 .
$$

For use in exterior domains one can also allow negative $n$ above. In general, we advise the reader to prefer harmonic polynomials in polar coordinates over those in Cartesian coordinates, though there is some bias towards the special role of the origin.

The next step considers real parts of power series

$$
P_{\Lambda}(z):=\sum_{n=0}^{\infty} \lambda_{n} z^{n}
$$

which are convergent in a disc of radius $\rho>0$ around zero and which have real coefficients. They lead to harmonic functions

$$
F_{\Lambda}(r, \varphi):=\sum_{n=0}^{\infty} \lambda_{n} r^{n} \cos (n \varphi)
$$

for all points $(r, \varphi)$ with $r<\rho$. 
To get a kernel which is harmonic in two 2D variables $(r, \varphi)$ and $(s, \psi)$ in polar coordinates, we can superimpose harmonic polynomials

$$
r^{n} \cos (n \varphi), r^{n} \sin (n \varphi), s^{m} \cos (m \psi), s^{m} \sin (m \psi)
$$

in an arbitrary way. Motivated by Mercer kernels from machine learning and by the above observation, we consider a special superposition

$$
\begin{aligned}
\left.K_{\Lambda, c}((r, \varphi) ;(s, \psi))\right) & :=\sum_{n=0}^{\infty} \lambda_{n} c^{2 n} r^{n} s^{n} \cos (n(\varphi-\psi)) \\
& =F_{\Lambda}\left(c^{2} r s, \varphi-\psi\right)
\end{aligned}
$$

to get a symmetric harmonic kernel under the summability condition

$$
\sum_{n=0}^{\infty} \lambda_{n} c^{2 n} r^{n} s^{n}<\infty
$$

with otherwise arbitrary positive weights $\lambda_{n}$ and a scaling factor $c>0$. Taking our domain into account, we shall strengthen the above condition to

$$
\sum_{n=0}^{\infty} \lambda_{n} c^{2 n} R^{2 n}<\infty
$$

in order to be able to evaluate safely on the boundary.

Theorem 1 Harmonic kernels of the above form are positive definite, and this also holds for their periodicized form.

Proof We look at the usual quadratic form for points $\left(r_{j}, \varphi_{j}\right), 1 \leq j \leq N$ and get the nonnegative result

$$
\begin{aligned}
\sum_{j, k=1}^{N} a_{j} a_{k} & \sum_{n=0}^{\infty} \lambda_{n} c^{2 n} r_{j}^{n} r_{k}^{n} \cos \left(n\left(\varphi_{j}-\varphi_{k}\right)\right) \\
& =\sum_{n=0}^{\infty} \lambda_{n} c^{2 n} \sum_{j, k=1}^{N} a_{j} a_{k} r_{j}^{n} r_{k}^{n} \cos \left(n\left(\varphi_{j}-\varphi_{k}\right)\right) \\
& =\sum_{n=0}^{\infty} \lambda_{n} c^{2 n}\left(\left(\sum_{j=1}^{N} a_{j} r_{j}^{n} \cos \left(n \varphi_{j}\right)\right)^{2}+\left(\sum_{j=1}^{N} a_{j} r_{j}^{n} \sin \left(n \varphi_{j}\right)\right)^{2}\right)
\end{aligned}
$$

If the form vanishes, we necessarily have

$$
\sum_{j=1}^{N} a_{j} r_{j}^{n} \exp \left(i n \varphi_{j}\right)=0
$$

for all $n \geq 0$, which means that $a$ is in the kernel of the infinite Vandermonde matrix with entries $z_{j}^{n}=r_{j}^{n} \exp \left(\operatorname{in} \varphi_{j}\right)$, but this matrix must have rank $N$ because polynomials of unlimited degree always separate points. This implies $a=0$ and 
positive definiteness. Clearly, the result extends to parameterized subsets of the domain.

\section{Special harmonic kernels}

This section will borrow from the univariate periodic kernels provided by Anette Meyenburg [4] in the early years of kernel techniques.

We start our construction of harmonic kernels with a very fast convergent case, setting $\lambda_{n}:=\frac{1}{n !}$ and using the entire exponential function $P(z)=\exp (z)$. The result is the analytic $2 \pi$-periodic kernel

$$
\begin{aligned}
\Re(\exp (z)) & =\Re \sum_{n=0}^{\infty} \frac{z^{n}}{n !} \\
& =\sum_{n=0}^{\infty} \frac{r^{n} \cos (n \varphi)}{n !} \\
\Re(\exp (z)) & =\Re(\exp (r \cos (\varphi)+i r \sin (\varphi))) \\
& =\exp (r \cos (\varphi)) \cdot \Re(\exp (i r \sin (\varphi))) \\
& =\exp (r \cos (\varphi)) \cdot \cos (r \sin (\varphi))
\end{aligned}
$$

which yields the globally harmonic "exponential” kernel

$$
\begin{aligned}
\left.K_{\Lambda, c}((r, \varphi) ;(s, \psi))\right) & :=\sum_{n=0}^{\infty} \frac{1}{n !} c^{2 n} r^{n} s^{n} \cos (n(\varphi-\psi)) \\
& =\exp \left(c^{2} r s \cos (\varphi-\psi)\right) \cdot \cos \left(c^{2} r s \sin (\varphi-\psi)\right) .
\end{aligned}
$$

Making $c$ large results in a sharp spike, but does not affect global summability.

If we want to generate a kernel from a meromorphic function, we can work with the geometric series to get the Poisson kernel

$$
\begin{aligned}
\Re\left(\frac{1}{1-z}\right) & =\Re \sum_{n=0}^{\infty} z^{n} \\
& =\sum_{n=0}^{\infty} r^{n} \cos (n \varphi) \\
\Re\left(\frac{1}{1-z}\right) & =\Re\left(\frac{1}{1-r \cos (\varphi)-i r \sin (\varphi)}\right) \\
& =\Re\left(\frac{1-r \cos (\varphi)+i r \sin (\varphi)}{(1-r \cos (\varphi))^{2}+r^{2} \sin ^{2}(\varphi)}\right) \\
& =\frac{1-r \cos (\varphi)}{1-2 r \cos (\varphi)+r^{2}}
\end{aligned}
$$


which requires $r<1$. This yields the harmonic kernel

$$
\begin{aligned}
\left.K_{\Lambda, c}((r, \varphi) ;(s, \psi))\right) & :=\sum_{n=0}^{\infty} c^{2 n} r^{n} s^{n} \cos (n(\varphi-\psi)) \\
& =\frac{1-c^{2} r s \cos (\varphi-\psi)}{1-2 c^{2} r s \cos (\varphi-\psi)+c^{4} r^{2} s^{2}} .
\end{aligned}
$$

Here, making $c$ small enough helps to satisfy the summability condition

$$
c^{2} r s<1,
$$

and a large value within this range will again result in a sharp spike. But since the second form of the kernel is the analytic continuation of the series form, we can drop the summability condition to find that the Poisson kernel can be used everywhere except for

$$
c^{2} r s=1 \text { and } \varphi-\psi \in 2 \pi \mathbb{Z}
$$

where it has a singularity.

\section{Singularities of the Poisson kernel}

When using the Poisson kernel, the implicit placement of the singularities becomes important. A single basis function written in a $2 \mathrm{D}$ variable in polar coordinates $(s, \psi)$ as

$$
g_{j}(s, \psi):=\frac{1-c^{2} \rho_{j} s \cos \left(t_{j}-\psi\right)}{1-2 c^{2} \rho_{j} s \cos \left(t_{j}-\psi\right)+c^{4} \rho_{j}^{2} s^{2}}
$$

for a fixed point $\mathcal{R}_{j}=\left(\rho\left(t_{j}\right), t_{j}\right)$ will have a singularity at the "reflected" point $\left(1 /\left(c^{2} \rho_{j}\right), t_{j}\right)$. Note that reflected points arise naturally in standard constructions of Green's functions for the Laplace operator [9]. The reflected points should lie outside the domain in question, and since we here consider the origin to be inside a starshaped domain with boundary $\{(\rho(t), t), t \in[0,2 \pi]\}$ in polar coordinates, we need

$$
\frac{1}{c^{2} \rho_{j}}>\rho_{j} \text { or } c<\frac{1}{\rho_{j}}
$$

to see that $c<1 / \rho_{j}$ will place the singularity outside the domain, while $c=1 / \rho_{j}$ lets the singularity coincide with our data point. To play safe, one must set

$$
c<\frac{1}{\max _{t} \rho(t)}
$$

to make sure that the resulting superposition of such functions on arbitrary boundary points does not produce singularities inside the domain.

If $c$ is fixed this way, each boundary point $(\rho(t), t)$ has a reflected point $\left(1 /\left(c^{2} \rho(t)\right), t\right)$, and the reflected points come close to the boundary only where $r$ takes its extremal values. This means that incoming corners of the domain cannot have close reflection points, and this will make it hard to work on such domains, as 

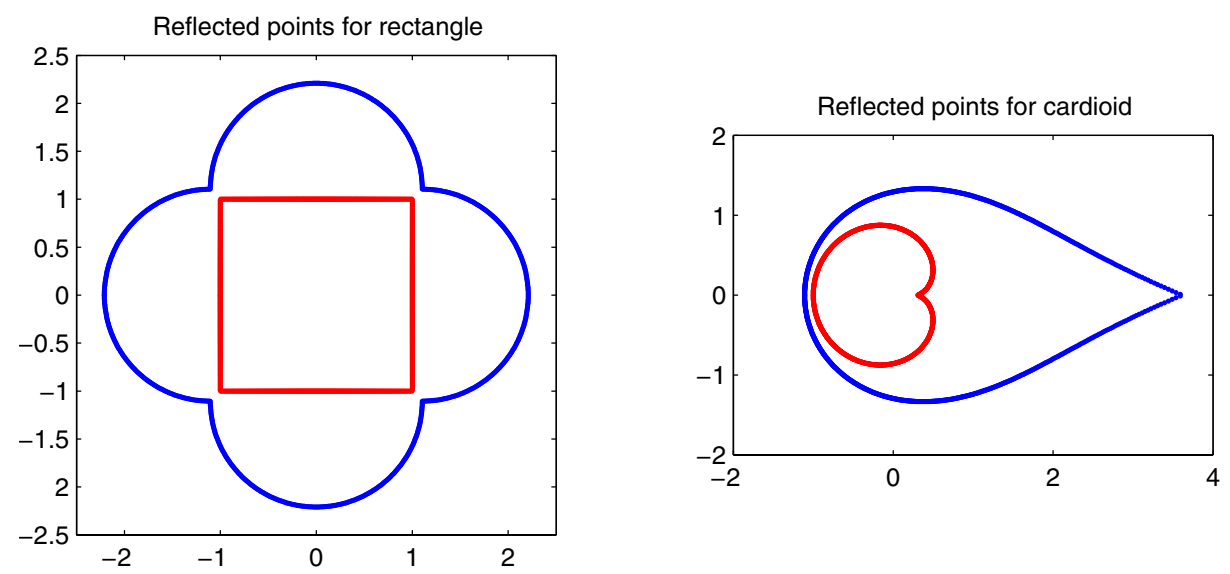

Fig. 1 Reflected boundary

we see later. Figure 1 shows the reflected points for both a rectangle and a cardioid. The constant $c$ was chosen to be

$$
c=\frac{0.95}{\max _{t} \rho(t)}
$$

in both cases. One can see how the singularities in the rectangle case come close to the corners, while they stay away from the cardioid's incoming corner.

For problems on exterior domains, one should of course choose

$$
c>\frac{1}{\min _{t} \rho(t)}
$$

to get a similar argument.

\section{Error bounds}

For error analysis, we first invoke a local univariate sampling inequality of the form

$$
\|u\|_{L_{\infty}[a, b]} \leq c h^{M-1 / 2}|u|_{W_{2}^{M}[a, b]}
$$

for functions $u$ in Sobolev space $W_{2}^{M}[a, b]$ with $M>1 / 2$ which vanish on a set $X$ of points in $[a, b]$ with fill distance

$$
h:=\sup _{x \in[a, b]} \min _{x_{j} \in X}\left\|x-x_{j}\right\| \leq h_{0},
$$

and the constant $c$ does only depend on the domain and the numbers $M, h_{0}$. See $[5,11]$ for details of such inequalities.

This inequality applies to errors of interpolation processes, provided that the Sobolev seminorm $|s|_{W_{2}^{M}[a, b]}$ of interpolants on $X$ can be bounded independent of the fill distance $h$, as is the case with splines and other kernel-based techniques minimizing certain norms or seminorms in certain "native" Hilbert spaces in which the kernels are reproducing. 
To apply these techniques, we have to prove that the native Hilbert space of the periodicized kernel $\tilde{K}$ is continuously embedded in a Sobolev space $W_{2}^{M}[a, b]$ of highest possible order. If we manage this for order $M$, we get a convergence rate like $h^{M-1 / 2}$ by the sampling inequality, and since this error bound extends to the interior via the Maximum Principle, it holds on the whole domain.

Finally we remark that the above analysis applies locally to parts of the boundary. Thus one can expect good convergence rates where there are no singularities induced by the boundary shape or the boundary data. This will be confirmed by examples.

\section{Smoothness results}

To make the above argument work, we have to investigate the smoothness of our trial functions more closely. We represent the $2 \pi$-periodic function

$$
\begin{aligned}
g(t) & :=\sum_{j=1}^{N} a_{j} \tilde{K}\left(t_{j}, t\right) \\
& =\sum_{j=1}^{N} a_{j} K\left(\rho\left(t_{j}\right), \rho(t)\right)
\end{aligned}
$$

as

$$
\begin{aligned}
g(t) & =\sum_{j=1}^{N} a_{j} \sum_{n=0}^{\infty} \lambda_{n} c^{2 n} \rho^{n}\left(t_{j}\right) \rho^{n}(t) \cos \left(n\left(t-t_{j}\right)\right) \\
& =\sum_{n=0}^{\infty} \lambda_{n} c^{2 n} \rho^{n}(t)\left(c_{n} \cos (n t)+d_{n} \sin (n t)\right) .
\end{aligned}
$$

with

$$
\begin{aligned}
c_{n} & :=\sum_{j=1}^{N} a_{j} \rho^{n}\left(t_{j}\right) \cos \left(n t_{j}\right) \\
d_{n} & :=\sum_{j=1}^{N} a_{j} \rho^{n}\left(t_{j}\right) \sin \left(n t_{j}\right) .
\end{aligned}
$$

In the native space for the periodicized kernel $\tilde{K}$, this function has the norm

$$
\begin{aligned}
\|g\|_{\tilde{K}}^{2} & =\sum_{j, k=1}^{N} a_{j} a_{k} \tilde{K}\left(t_{j}, t_{k}\right) \\
& =\sum_{j, k=1}^{N} a_{j} a_{k} \sum_{n=0}^{\infty} \lambda_{n} c^{2 n} \rho\left(t_{j}\right)^{n} \rho\left(t_{k}\right)^{n} \cos \left(n\left(t_{k}-t_{j}\right)\right) \\
& =\sum_{n=0}^{\infty} \lambda_{n} c^{2 n}\left(c_{n}^{2}+d_{n}^{2}\right)
\end{aligned}
$$


which is finite due to our summability condition. To arrive at error bounds in Sobolev spaces, we have to bound derivatives of $g$ in terms of this norm. We assume the boundary function $\rho$ to be $k$-fold continuously differentiable. Then all functions of the form of $g$ are $k$-fold continuously differentiable. To bound derivatives of $g$ from above, we have to bound derivatives of the functions

$$
\rho^{n}(t) \cos (n t) \text { and } \rho^{n}(t) \sin (n t)
$$

from above, and we have to care for the behavior with respect to large $n$. The $j$-th derivative of $\rho^{n}$ has $n^{j}$ bounded terms, and then the product rule shows that the $k$-th derivative of the above functions is bounded by a constant times $2^{k} n^{k}$. Then we get

$$
\begin{aligned}
g^{(k)}(t) & =\sum_{n=0}^{\infty} \lambda_{n} c^{2 n}\left(c_{n}\left(\rho^{n}(t) \cos (n t)\right)^{(k)}+d_{n}\left(\rho^{n}(t) \sin (n t)\right)^{(k)}\right) \\
\left|g^{(k)}(t)\right| & \leq C 2^{k} \sum_{n=0}^{\infty} \lambda_{n} c^{2 n} n^{k} \sqrt{c_{n}^{2}+d_{n}^{2}} \\
& \leq C 2^{k} \sqrt{\sum_{n=0}^{\infty} \lambda_{n} c^{2 n}\left(c_{n}^{2}+d_{n}^{2}\right)} \sqrt{\sum_{n=0}^{\infty} \lambda_{n} c^{2 n} n^{2 k}}
\end{aligned}
$$

and

$$
\|g\|_{W_{2}^{k}[0,2 \pi]}^{2} \leq C\|g\|_{\tilde{K}}^{2} 4^{k} \sum_{n=0}^{\infty} \lambda_{n} c^{2 n} n^{2 k} .
$$

The constant $C$ above is formally independent of $k$, but contains a uniform bound of all derivatives of the boundary function up to order $k$.

Theorem 2 If the boundary function is in $C^{k}$ and if

$$
c_{k}^{2}:=C 4^{k} \sum_{n=0}^{\infty} \lambda_{n} c^{2 n} n^{2 k}<\infty,
$$

then the native space of the periodicized kernel $\tilde{K}$ is continuously embedded in Sobolev space $W_{2}^{k}[0,2 \pi]$ with norm $c_{k}$ of the embedding. Furthermore, interpolation by harmonic kernels on the boundary has an error of order $h^{k-1 / 2}$ in the $L_{\infty}$ norm.

\section{Special cases}

If we take the exponential kernel, there is no summability condition on the kernel parameters to be satisfied, because convergence is guaranteed for all parameter choices. Consequently, the convergence rate is entirely determined by the smoothness of the domain and the smoothness of the data function. For domains with smoothness $C^{k}$ in polar parametrization, there always is convergence of order $k-1 / 2$ for smooth enough data, because the condition in Theorem 2 is satisfied for all parameter choices. For very smooth domains, i.e. when there is arbitrary smoothness order $k$ with $k$-independent bounds on the derivatives, the convergence rate is arbitrarily high, provided that the data function is of arbitrary smoothness. This occurs for 
circles or ellipses, for instance. By invoking sampling inequalities for infinitely differentiable functions [6], one can get exponential convergence rates, because the constants in Theorem 2 are uniformly bounded with respect to $k$. However, all of this is limited by the smoothness of the data function.

For the Poisson-type kernel, we saw that we have to be more careful. If we take (2), everything works. However, the condition of Theorem 2 is satisfied for all $k$, but not uniformly for $k \rightarrow \infty$. Thus the convergence rate, depending on the smoothness of the domain and the data function, can be arbitrarily high but is not necessarily exponential.

\section{Numerical results}

We ran a series of test examples, and Table 1 contains some typical results we shall explain now. After quite some experiments, we settled for choosing

$$
c=\frac{0.85}{\max _{t} \rho(t)}
$$

in view of (2) for the Poisson kernel, while we used

$$
c=\frac{1}{\max _{t} \rho(t)}
$$

for the exponential kernel, though there are no singularities to care for. The notations for the columns of Table 1 are as follows:

Table 1 Results of test runs

\begin{tabular}{llllrl}
\hline Dom & Fun & Ker & $N$ & $n$ & $\epsilon_{\infty}$ \\
\hline circ & y & exp & 200 & 24 & $7.622044 \mathrm{e}-010$ \\
circ & y & poi & 200 & 121 & $3.422198 \mathrm{e}-009$ \\
circ & poly & exp & 200 & 27 & $7.697248 \mathrm{e}-010$ \\
circ & poly & poi & 200 & 108 & $3.593031 \mathrm{e}-009$ \\
circ & abs & exp & 200 & 27 & $4.869433 \mathrm{e}-002$ \\
circ & abs & poi & 200 & 184 & $4.738652 \mathrm{e}-003$ \\
rect & poly & exp & 200 & 25 & $1.448961 \mathrm{e}-002$ \\
rect & poly & poi & 200 & 74 & $6.848998 \mathrm{e}-004$ \\
rect & abs & exp & 200 & 25 & $7.693859 \mathrm{e}-002$ \\
rect & abs & poi & 200 & 78 & $2.498604 \mathrm{e}-002$ \\
card & poly & exp & 200 & 25 & $1.286584 \mathrm{e}-003$ \\
card & poly & poi & 200 & 97 & $7.384259 \mathrm{e}-004$ \\
card & abs & exp & 200 & 24 & $2.175175 \mathrm{e}-001$ \\
card & abs & poi & 200 & 98 & $1.764641 \mathrm{e}-001$ \\
card & abs & poi & 200 & 124 & $7.695109 \mathrm{e}-002$ \\
\hline
\end{tabular}


Fig. 2 Point configuration for the last line of Table 1

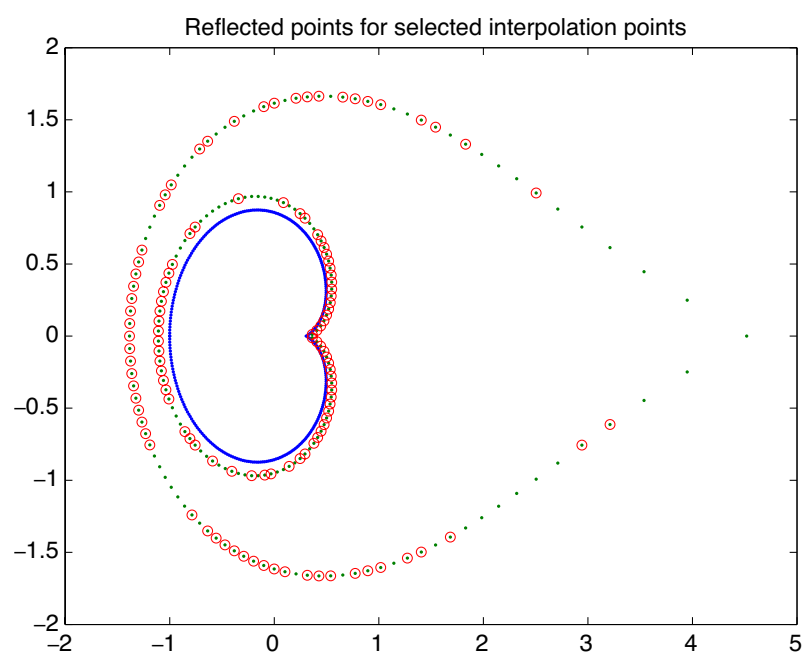

Dom = Domain, can be circle, rectangle or cardioid

$$
\rho(t)=\sqrt{c^{2}+|\sin (t / 2)|} / \sqrt{c^{2}+1}, c=0.3218
$$

Fun $\quad=$ Function $f(x, y)$, can be $\mathrm{y}=y$, poly $=y^{3} x^{2}$, abs $=|y|$

Ker $=$ Kernel, can be exp $=$ exponential or poi $=$ Poisson kernel

$N \quad=$ number of data points for collocation on boundary

$n \quad=$ number of selected basis functions, $=$ degrees of freedom used

$\epsilon_{\infty} \quad=L_{\infty}$ error on 1000 test points on boundary

The algorithm used for providing the examples is a variant of the adaptive technique presented in [7] which selects a certain subset of the collocation data locations for use as trial points in a representation (1). In the table, $N$ stands for the full

Fig. 3 Error behavior for the last line of Table 1
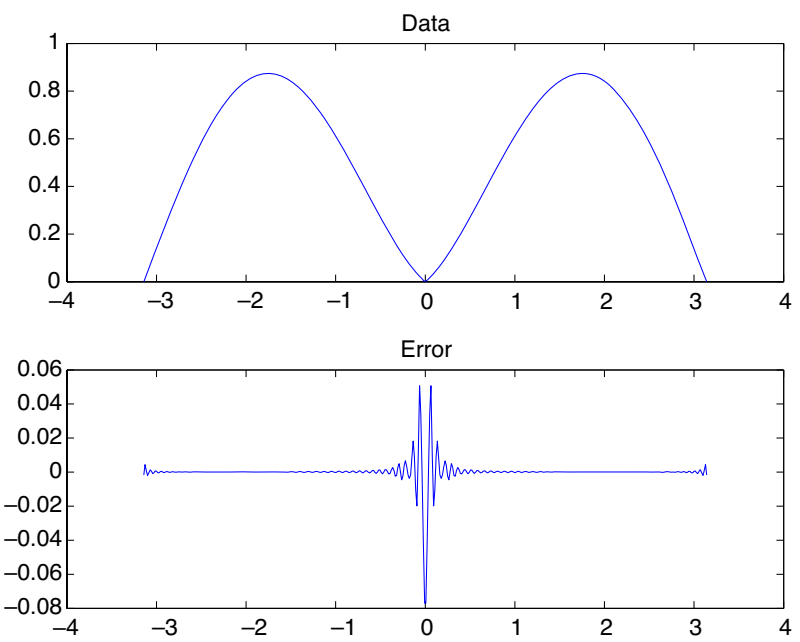
number of data and collocation points, of which $n$ are adaptively selected for entering into a representation (1) on the trial side. The selection is stopped when either a relative improvement of 0.00001 or an absolute improvement by at least the machine precision is impossible. A MATLAB ${ }^{\odot}$ program reproducing all examples is available from the author's website.

Users should be aware that the exponential kernel will work best if the data come from a globally harmonic singularity-free function. This occurs for the first two lines of Table 1. Since the solution is singularity-free, the Poisson kernel needs 121 degrees of freedom, while the exponential kernel uses only 24 .

The next two lines of Table 1 show a case interpolating non-harmonic data from $f(x, y)=x^{2} y^{3}$ on the unit circle. This fits into our error analysis, and the rate of approximation should be exponential. Again, the exponential kernel is superior.

We no go to the rectangle. By our theory, this induces nondifferentiability into the problem, even if the data are smooth like poly $=f(x, y)=x^{2} y^{3}$. The error for the Poisson kernel now gets better, because due to Fig. 1 the singularities of the Poisson kernel come close to the corners. If we take $f(x, y)=|y|$ on the rectangle, we get two additional derivative singularities on smooth parts of the boundary, but these cannot be reached by singularities of the Poisson kernel due to Fig. 1. Consequently, its performance drops.
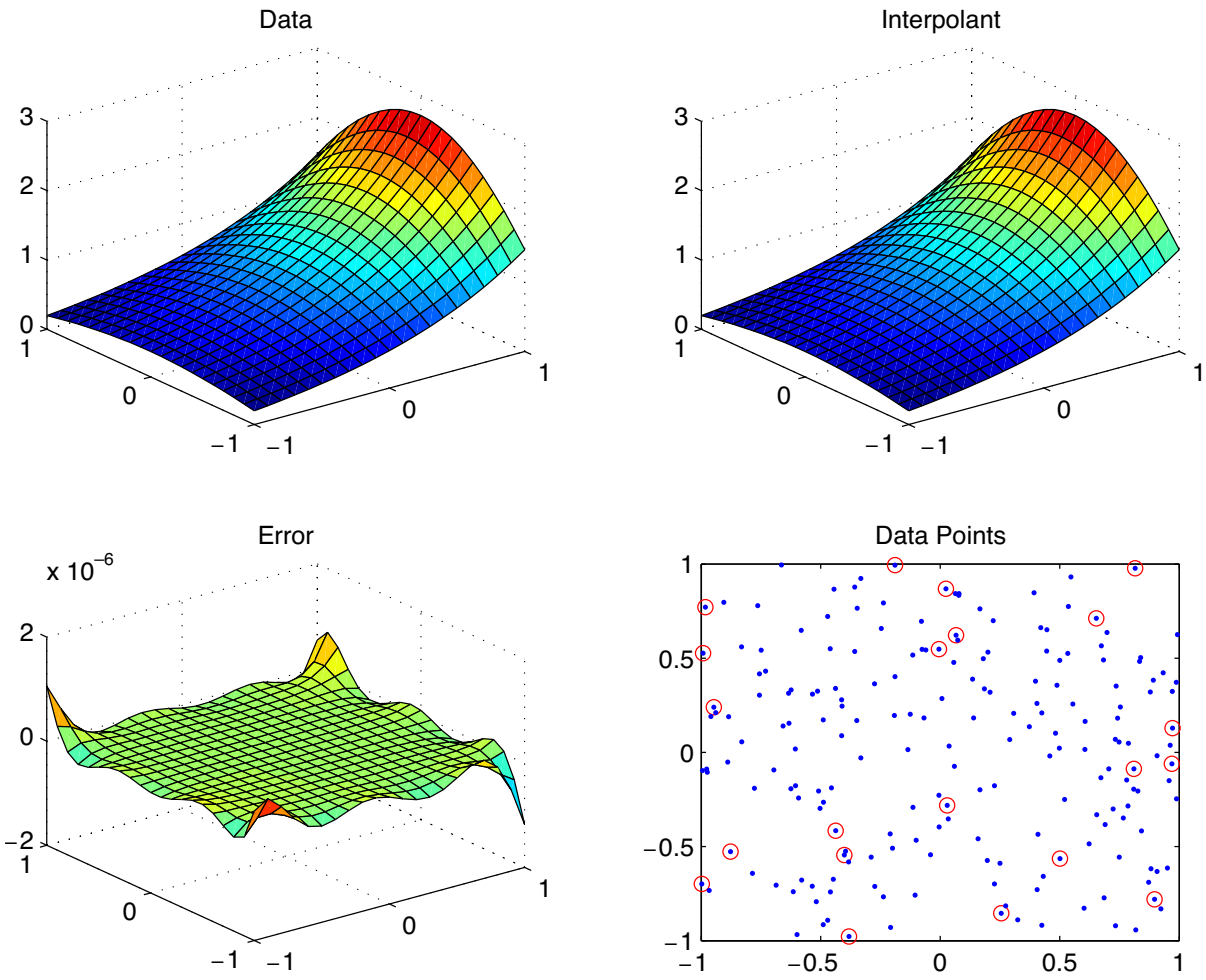

Fig. 4 Recovery of the harmonic function $f(x, y)=y$ from scattered data, using the exponential kernel 
On the cardioid, we cannot reach the incoming corner by singularities in reflected points, due to Fig. 1. Even for smooth data, all algorithms have problems. But this also occurs for other methods like the MFS, unless special singularity-dependent trial functions are added manually.

Now for the separate final line of Table 1. There, we treated 100 points on the boundary as before, but the other 100 got close-by reflection points by choosing a point-dependent $c$ according to the rule

$$
c\left(t_{j}\right)=\frac{0.95}{\rho\left(t_{j}\right)} .
$$

The 200 offered and 124 adaptively selected points are shown in Fig. 2. Note that our convergence analysis still works, because we kept half of the points in the standard setting, adding certain additional degrees of freedom which can only decrease the error. Figure 3 shows the error behavior. Since our convergence analysis is completely local, the error is everywhere small except near the singularities. Similar results are obtained also in the other cases we treated before: the bad error behavior is confined to neighborhoods of the singularities.

But one can also be as bold as to fit harmonic functions to scattered data. This is an ill-posed problem, but algorithmically it will work, even for general nonharmonic functions. However, due to the maximum principle, the error behavior
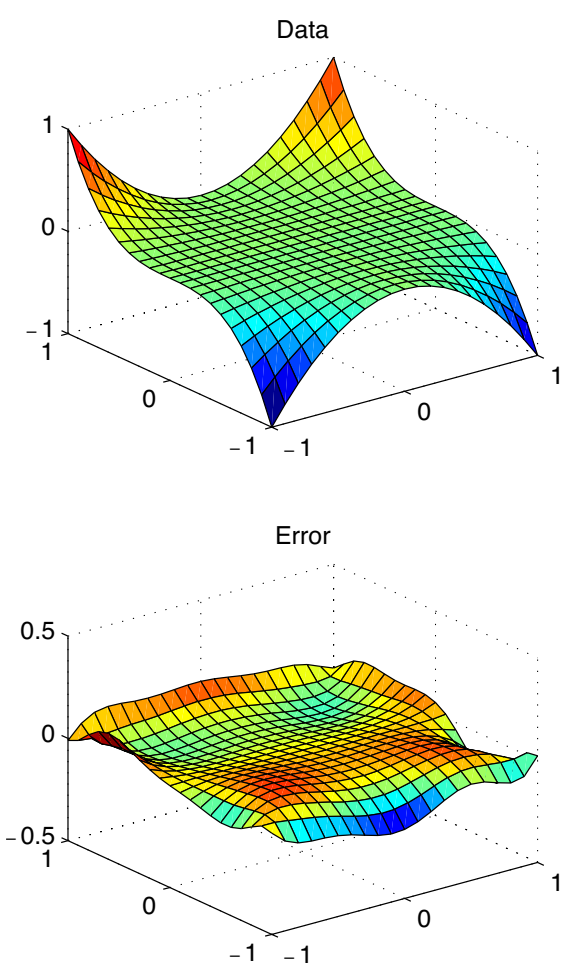
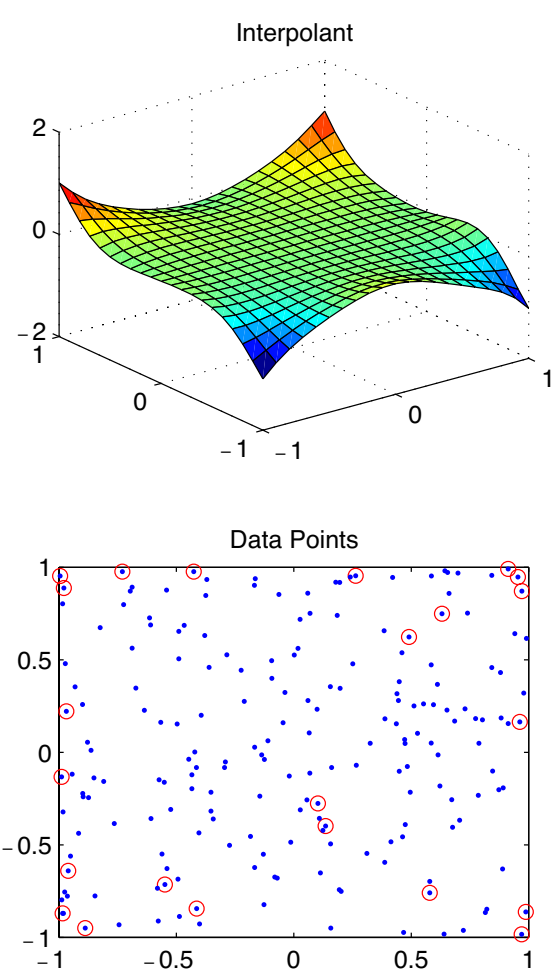

Fig. 5 Harmonic interpolation of scattered data provided by the non-harmonic function $y^{3} x^{2}$, using the exponential kernel 
will be disastrous if the data are not close to those of a harmonic function. Figure 4 shows the well-posed recovery of a harmonic function from its scattered data, while Fig. 5 shows how our kernel method tries to do the impossible: providing a harmonic interpolant to data coming from a non-harmonic function.

\section{Conclusions}

This paper seems to be the first to produce a fairly general quantitative error analysis for a method which solves homogeneous boundary-value problems by a simple superposition of homogeneous solutions centered at boundary points. In contrast to the method of fundamental solutions, no "fictitious" or "artificial" boundary is needed, and the convergence analysis works for general domains.

The method should be generalized to other differential operators, and possibly applied to inverse problems. In general, it seems to be a promising strategy to use kernel techniques which are tailored to solve certain PDE problems, using newly designed kernels.

Open Access This article is distributed under the terms of the Creative Commons Attribution Noncommercial License which permits any noncommercial use, distribution, and reproduction in any medium, provided the original author(s) and source are credited.

\section{References}

1. Cho, H.A., Golberg, M.A., Muleshkov, A.S., Li, X.: Trefftz methods for time dependent partial differential equations. Computers, Materials, and Continua 1, 1-38 (2004)

2. Fairweather, G., Karageorghis, A.: The method of fundamental solution for elliptic boundary value problems. Adv. Comput. Math. 9, 69-95 (1998)

3. Golberg, M.A., Chen, C.S.: The method of fundamental solutions for potential, Helmholtz and diffusion problems. In: Golberg, M.A. (ed.) Boundary Integral Methods: Numerical and Mathematical Aspects, pp. 103-176. WIT Press (1998)

4. Meyenburg, A.: Interpolation mit Translaten einer geraden $2 \pi$-periodischen stetigen Funktion. Master's thesis, Universität Göttingen, Staatsexamensarbeit NAM-E-390 (1996)

5. Narcowich, F.J., Ward, J.D., Wendland, H.: Sobolev bounds on functions with scattered zeros, with applications to radial basis function surface fitting. Math. Comput. 74, 743-763 (2003)

6. Rieger, C., Zwicknagl, B.: Sampling inequalities for infinitely smooth functions, with applications to interpolation and machine learning. Preprint Göttingen/Leipzig, Journal of Machine Learning Research (2008, to appear)

7. Schaback, R.: Adaptive numerical solution of MFS systems. (2007, preprint)

8. Schaback, R., Wendland, H.: Kernel techniques: from machine learning to meshless methods. Acta Numerica 15, 543-639 (2006)

9. Sommerfeld, A.: Partial Differential Equations in Physics. Academic Press, New York (1949)

10. Wendland, H.: Scattered Data Approximation. Cambridge Monographs on Applied and Computational Mathematics. Cambridge University Press, Cambridge (2004)

11. Wendland, H., Rieger, C.: Approximate interpolation with applications to selecting smoothing parameters. Numer. Math. 101, 643-662 (2005) 